\title{
Positive solutions to P-Laplacian multi-point BVPs on time scales
}

\author{
Subei Li, You-hui Su, and Zhaosheng Feng \\ Communicated by $Y$. Charles Li, received December 23, 2009.
}

\begin{abstract}
In this paper, we are concerned with the $p$-Laplacian multi-point boundary value problem with the first-order derivative of the nonlinear term on time scales. Some new sufficient conditions are presented for the existence of at least twin or arbitrary even positive solutions to the multi-point boundary value problem of the $p$-Laplacian dynamic equation on time scales. In particular, our results are even new for some special cases of differential and difference equations in the general time scale setting. As an application, an example is illustrated at the end of the article.
\end{abstract}

\section{Contents}

1. Introduction 45

2. Some Lemmas 48

3. Existence Results of Problem (1.4) and (1.5) 51

4. Existence Results of Problem (1.4) and (1.6) 59

5. Example 61

References $\quad 63$

\section{Introduction}

In the past few decades, the theory of time scales remains a field of vitality and receives a lot of attention from a rather diverse group of scientists such as physicists and mathematicians, because it can not only unify differential and difference equations $[\mathbf{1}, \mathbf{6}, \mathbf{7}, \mathbf{1 4}]$, but also provide the accurate information of phenomena that

1991 Mathematics Subject Classification. Primary 34B15, 35B09; Secondary 39A10.

Key words and phrases. Time scales, boundary value problem, positive solutions, $p$ Laplacian, fixed point theorem.

This work is supported by the Grant of Department of Education of Jiangsu Province under 09KJD110006.

This work is partially supported by UTPA Faculty Research Council Grant 119100. 
manifest themselves partly in continuous time and partly in discrete time. Nowadays the theory of time scales has been widely applied to biology, heat transfer, stock market, wound healing and epidemic models $[\mathbf{5}, \mathbf{8}, \mathbf{2 0}]$, etc.

Recently, positive solutions of boundary value problems (BVPs) for dynamic equations on time scales were studied intensively with various conditions (see [9, $10,11,12,13,22,23,24,25,26,27,28,29]$ and the references therein). In [28], Sun and Li considered the two-point BVPs on time scales of form

$$
\begin{gathered}
\left(\varphi_{p}\left(u^{\Delta}(t)\right)\right)^{\Delta}+h(t) f\left(u^{\sigma}(t)\right)=0, \quad t \in[a, b]_{\mathbb{T}}, \\
u(a)-B_{0}\left(u^{\Delta}(a)\right)=0, \quad u^{\Delta}(\sigma(b))=0 .
\end{gathered}
$$

The existence theory for positive solutions of the above BVPs on time scales by using the fixed-point theorem in cone $[\mathbf{3}, \mathbf{1 6}, \mathbf{1 8}]$.

In [24], $\mathrm{Su}$ and Li considered the multi-point BVPs on time scale of form

$$
\begin{gathered}
\left(\varphi_{p}\left(u^{\Delta}(t)\right)\right)^{\nabla}+h(t) f(u(t))=0, \quad t \in[0, T]_{\mathbb{T}}, \\
u(0)-B_{0}\left(\sum_{i=1}^{m-2} a_{i} u^{\Delta}\left(\xi_{i}\right)\right)=0, \quad u^{\Delta}(T)=0,
\end{gathered}
$$

or

$$
u^{\Delta}(0)=0, \quad u(T)+B_{1}\left(\sum_{i=1}^{m-2} b_{i} u^{\Delta}\left(\xi_{i}^{\prime}\right)\right)=0,
$$

and obtained the existence criteria for at least three positive solutions of problem (1.1) and (1.2) or (1.3) by using a generalization of the Leggett-Williams fixed point theorem $[4]$.

It is noted that the works mentioned above $[\mathbf{9}, \mathbf{1 0}, \mathbf{1 1}, \mathbf{1 2}, \mathbf{1 3}, \mathbf{2 2}, \mathbf{2 3}, \mathbf{2 4}$, $\mathbf{2 5}, \mathbf{2 6}, \mathbf{2 7}, \mathbf{2 8}, \mathbf{2 9}$ ] did not consider the existence of positive solutions to BVPs on time scales with the nonlinear term $f$ involving the first-order derivative. To the best of our knowledge so far, it seems that there is very few literature regarding the existence of positive solution to BVPs on time scales when the nonlinear function $f$ contains the derivative term. In $[\mathbf{2 1}]$ we only dealt with positive solutions to a singular $p$-Laplacian BVP with sign-changing nonlinearity involving derivative on time scales by using the upper and lower solution method, but the location and character of positive solutions was not analyzed. In this paper, we will not only investigate the existence of positive solutions, but also give the location and character of positive solutions. For the $p$-Laplacian multi-point BVPs on time scales, it is quite natural and necessary to consider the existence of positive solutions for the $p$-Laplacian multi-point BVPs on time scales with the nonlinear term $f$ involving the first-order derivative in all respects.

Motivated by our early works $[\mathbf{2 1}, \mathbf{2 2}, \mathbf{2 3}, \mathbf{2 4}, \mathbf{2 5}, \mathbf{2 6}]$, now we all-sidedly consider the following BVPs on time scales of form

$$
\left(\varphi_{p}\left(u^{\Delta}(t)\right)\right)^{\nabla}+h(t) f\left(t, u(t), u^{\Delta}(t)\right)=0, t \in[0, T]_{\mathbb{T}},
$$

subject to the multi-point boundary conditions

or

$$
u(0)-B_{0}\left(\sum_{i=1}^{m-2} a_{i} u^{\Delta}\left(\xi_{i}\right)\right)=0, u^{\Delta}(T)=0
$$

$$
u^{\Delta}(0)=0, u(T)+B_{1}\left(\sum_{i=1}^{m-2} b_{i} u^{\Delta}\left(\xi_{i}^{\prime}\right)\right)=0
$$

where $\xi_{i}, \xi_{i}^{\prime} \in[0, T]_{\mathbb{T}}$, and satisfy $0 \leq \xi_{1}<\xi_{2}<\ldots<\xi_{m-2}<\rho(T), \sigma(0)<\xi_{1}^{\prime}<$ $\xi_{2}^{\prime}<\ldots<\xi_{m-2}^{\prime} \leq T, a_{i}, b_{i} \in[0, \infty)(i=1,2, \ldots, m-2)$. Some novel theoretical 
results are obtained for the existence of at least twin or arbitrary even positive solutions of the above problem by using the fixed point theorem due to Avery and Henderson [3]. Notice that when $f\left(t, u, u^{\Delta}\right)=f(u)$, our results include the existing results of $[\mathbf{1 0}, \mathbf{1 2}, \mathbf{1 1}, \mathbf{1 9}]$ as particular cases for $\mathbb{T}=\mathbb{Z}$. Our results are even new for the special cases of difference equations and differential equations in the general time scale setting $[\mathbf{6}, \mathbf{7}]$.

Throughout the remainder of the paper, we denote by $\mathbb{T}$ a closed nonempty subset of $\mathbb{R}$, and let $\mathbb{T}$ have the subspace topology inherited from the Euclidean topology on $\mathbb{R}$. In some of current literature, $\mathbb{T}$ is the so-called a time scale. For our convenience, we make the blanket assumption that 0 and $T$ are two points in $\mathbb{T}$, and for an interval $(0, T)_{\mathbb{T}}$ we always mean $(0, T) \cap \mathbb{T}$. Other types of intervals are defined in the same way. We denote the $p$-Laplacian operator by $\varphi_{p}(u)$, i.e., $\varphi_{p}(u)=|u|^{p-2} u, p>1,\left(\varphi_{p}\right)^{-1}=\varphi_{q}, 1 / p+1 / q=1$. In addition, $B_{0}$ and $B_{1}$ satisfy

$$
A^{\prime} x \leq B_{i}(x) \leq B x, \quad x \in \mathbb{R}^{+}, \quad i=0,1,
$$

where $A^{\prime}$ and $B$ are positive real numbers.

We note that for a solution $u(t)$ of problems (1.4) and (1.5) or (1.6), we mean $u(t): \mathbb{T} \rightarrow \mathbb{R}$ which is the delta differential, $u^{\Delta}(t)$ and $\left(\varphi_{p}\left(u^{\Delta}\right)\right)^{\nabla}(t)$ are both continuous on $\mathbb{T}^{\kappa} \cap \mathbb{T}_{\kappa}$, and $u(t)$ satisfies problems (1.4) and (1.5) or (1.6). If $\left(u^{\Delta}\right)^{\nabla}(t) \leq 0$, then we say $u(t)$ is concave on $[0, T]_{\mathbb{T}}$.

In order to present our results in a straightforward manner and make this paper be fully self-contained, here we introduce some basic definitions which can be found in $[6,7]$. Alternative source on dynamical systems on measure chains is Reference [17].

Definition 1.1. A time scale $\mathbb{T}$ is a nonempty closed subset of $\mathbb{R}$. For $t \in \mathbb{T}$, the forward and back jump operators $\sigma, \rho: \mathbb{T} \rightarrow \mathbb{T}$ are well defined, respectively, by

$$
\sigma(t)=\inf \{s \in \mathbb{T}: s>t\} \text { and } \rho(t)=\sup \{s \in \mathbb{T}: s<t\} .
$$

In this definition, one puts $\inf \emptyset:=\sup \mathbb{T}$ and $\sup \emptyset:=\inf \mathbb{T}$, where $\emptyset$ denotes the empty set. A point $t \in \mathbb{T}$ is called left-dense if $\rho(t)=t$, left-scattered if $\rho(t)<t$, right-dense if $\sigma(t)=t$, and right-scattered if $\sigma(t)>t$. If $\mathbb{T}$ has a right-scattered minimum $m$, we define $\mathbb{T}_{\kappa}=\mathbb{T}-\{m\}$; otherwise, we set $\mathbb{T}_{\kappa}=\mathbb{T}$. If $\mathbb{T}$ has a leftscattered maximum $M$, we define $\mathbb{T}^{\kappa}=\mathbb{T}-\{M\}$; otherwise, we set $\mathbb{T}^{\kappa}=\mathbb{T}$. The forward graininess is $\mu(t):=\sigma(t)-t$, and the backward graininess is $\nu(t):=t-\rho(t)$.

Definition 1.2. If $f: \mathbb{T} \rightarrow \mathbb{R}$ is a function and $t \in \mathbb{T}^{\kappa}$, then the delta derivative of $f$ at the point $t$ is defined to the number $f^{\Delta}(t)$ (provided it exists) with the property that for any $\epsilon>0$, there is a neighborhood $U \subset \mathbb{T}$ of $t$ such that

$$
\left|f(\sigma(t))-f(s)-f^{\Delta}(t)(\sigma(t)-s)\right| \leq \epsilon|\sigma(t)-s| \text { for all } s \in U \text {. }
$$

If $f: \mathbb{T} \rightarrow \mathbb{R}$ and $t \in \mathbb{T}_{\kappa}$, then the nabla derivative of $f$ at the point $t$ is defined by the number $f^{\nabla}(t)$ (provided it exists) with the property that for any $\epsilon>0$, there is a neighborhood $U \subset \mathbb{T}$ of $t$ such that

$$
\left|f(\rho(t))-f(s)-f^{\nabla}(t)(\rho(t)-s)\right| \leq \epsilon|\rho(t)-s| \text { for all } s \in U .
$$

Definition 1.3. A function $f: \mathbb{T} \rightarrow \mathbb{R}$ is ld-continuous provided it is continuous at left dense points in $\mathbb{T}$ and its right sided limit exists (finite) at right dense points in $\mathbb{T}$.

Throughout this paper, we also assume that 
(S1): $f:[0, T]_{\mathbb{T}} \times \mathbb{R}^{+} \times \mathbb{R} \rightarrow \mathbb{R}^{+}$is ld-continuous, and does not vanish identically on any closed subinterval of $[0, T]_{\mathbb{T}}$, where $\mathbb{R}^{+}$denotes the nonnegative real numbers;

(S2): $h: \mathbb{T} \rightarrow \mathbb{R}^{+}$is left dense continuous (i.e., $h \in C_{l d}\left(\mathbb{T}, \mathbb{R}^{+}\right)$), and does not vanish identically on any closed subinterval of $[0, T]_{\mathbb{T}}$, where $C_{l d}\left(\mathbb{T}, \mathbb{R}^{+}\right)$ denotes the set of all left dense continuous functionals from $\mathbb{T}$ to $\mathbb{R}^{+}$;

(S3): While discussing problem (1.4) and (1.5), we suppose that if $\xi_{m-2}>0$, then let $0<\eta=\xi_{m-2}$; if $\xi_{m-2}=0$, then let $\eta=\min \left\{t \in \mathbb{T}: t \geq \frac{T}{2}\right\}$, and there exists $r \in \mathbb{T}$ such that $\eta<r<T$ holds. While discussing problem (1.4) and (1.6), we suppose that if $\xi_{1}^{\prime}<T$, then let $\xi=\xi_{1}^{\prime}$; if $\xi_{1}^{\prime}=T$, then let $\xi=\max \left\{t \in \mathbb{T}: 0<t \leq \frac{T}{2}\right\}$, and there exists $l \in \mathbb{T}$ such that $0<l<\xi<T$ holds.

The paper is organized as follows. In Section 2 we present some technical lemmas and fixed point theorems which we will use in the proofs of our main results. In Section 3, we will discuss the existence of at least twin or arbitrary even positive solutions to problem (1.4) and (1.5) under certain conditions. In Section 4 , sufficient conditions on the existence of positive solutions of problem (1.4) and (1.6) are established. Section 5 presents an example as application.

\section{Some Lemmas}

In this section, we introduce some preliminary theory of cones in Banach spaces [15], and then we state the fixed point theorems which we need in the proofs of our results in the next two sections.

Definition 2.1. Let $E$ be a real Banach space. A nonempty, closed, convex set $P \subset E$ is said to be a cone provided the following two conditions are satisfied:

(i) if $x \in P$ and $\lambda \geq 0$, then $\lambda x \in P$;

(ii) if $x \in P$ and $-x \in P$, then $x=0$. $P$.

Every cone $P \subset E$ induces an ordering in $E$ given by $x \leq y$ if and only if $y-x \in$

Definition 2.2. Given a cone $P$ in a real Banach space $E$, a functional $\psi$ : $P \rightarrow \mathbb{R}$ is said to be increasing on $P$, provided $\psi(x) \leq \psi(y)$ for all $x, y \in P$ with $x \leq y$.

Definition 2.3. A map $\alpha$ is said to be a nonnegative continuous concave functional on a cone $P$ of a real Banach space $E$ if $\alpha: P \rightarrow[0, \infty)$ is continuous and

$$
\alpha(t x+(1-t) y) \geq t \alpha(x)+(1-t) \alpha(y)
$$

for all $x, y \in P$ and $t \in[0,1]$. Similarly, we say the map $\beta$ is a nonnegative continuous convex functional on a cone $P$ of a real Banach space $E$ if $\beta: P \rightarrow[0, \infty)$ is continuous and

$$
\beta(t x+(1-t) y) \leq t \beta(x)+(1-t) \beta(y)
$$

for all $x, y \in P$ and $t \in[0,1]$.

In order to prove our main results, we also need the following three Lemmas:

LEMMA 2.4. [15] Let $P$ be a cone in a real Banach space. E and $U$ be a bounded, relatively open subset of $P$. Suppose that $A: \bar{U} \rightarrow P$ is a completely continuous 
operator and there exists $a u_{0}$ such that $x-A x \neq \lambda u_{0}$ for all $x \in \partial U$ and $\lambda \geq 0$. Then $i(A, U, P)=0$.

Given a nonnegative continuous functional $\psi$ on a cone $P$ of a real Banach space $E$, we define, for each $d>0$, the set

$$
P(\gamma, d)=\{x \in P: \gamma(x)<d\} .
$$

LEMma 2.5. [3] Let $P$ be a cone in a real Banach space E. Let $\alpha$ and $\gamma$ be increasing, nonnegative continuous functional on $P$, and let $\theta$ be a nonnegative continuous functional on $P$ with $\theta(0)=0$ such that, for some $c>0$ and $H>0$,

$$
\gamma(x) \leq \theta(x) \leq \alpha(x) \text { and }\|x\| \leq H \gamma(x) \text { for all } x \in \overline{P(\gamma, c)} \text {. }
$$

Suppose that there exists a completely continuous operator $A: \overline{P(\gamma, c)} \rightarrow P$ and $0<a<b<c$ such that

$$
\theta(\lambda x) \leq \lambda \theta(x) \text { for } 0 \leq \lambda \leq 1 \text { and } x \in \partial P(\theta, b),
$$

and

(i) $\gamma(A x)>c$ for all $x \in \partial P(\gamma, c)$;

(ii) $\theta(A x)<b$ for all $x \in \partial P(\theta, b)$;

(iii) $P(\alpha, a) \neq \emptyset$ and $\alpha(A x)>a$ for $x \in \partial P(\alpha, a)$.

Then $A$ has at least two fixed points $x_{1}$ and $x_{2}$ belonging to $\overline{P(\gamma, c)}$ satisfying

$$
a<\alpha\left(x_{1}\right) \text { with } \theta\left(x_{1}\right)<b \text { and } b<\theta\left(x_{2}\right) \text { with } \gamma\left(x_{2}\right)<c \text {. }
$$

Lemma 2.6. [19] Let $P$ be a cone in a real Banach space E. Let $\alpha$ and $\gamma$ be increasing, nonnegative continuous functional on $P$, and let $\theta$ be a nonnegative continuous functional on $P$ with $\theta(0)=0$ such that, for some $c>0$ and $H>0$,

$$
\gamma(x) \leq \theta(x) \leq \alpha(x) \text { and }\|x\| \leq H \gamma(x) \text { for all } x \in \overline{P(\gamma, c)} .
$$

Suppose there exist a completely continuous operator $A: \overline{P(\gamma, c)} \rightarrow P$ and $0<a<$ $b<c$ such that

$$
\theta(\lambda x) \leq \lambda \theta(x) \text { for } 0 \leq \lambda \leq 1 \text { and } x \in \partial P(\theta, b)
$$

and

(i) $\gamma(A x)<c$ for all $x \in \partial P(\gamma, c)$;

(ii) $\theta(A x)>b$ for all $x \in \partial P(\theta, b)$;

(iii) $P(\alpha, a) \neq \emptyset$ and $\alpha(A x)<a$ for $x \in \partial P(\alpha, a)$.

Then $A$ has at least two fixed points $x_{1}$ and $x_{2}$ belonging to $\overline{P(\gamma, c)}$ satisfying

$$
a<\alpha\left(x_{1}\right) \text { with } \theta\left(x_{1}\right)<b \text { and } b<\theta\left(x_{2}\right) \text { with } \gamma\left(x_{2}\right)<c \text {. }
$$

Although the statement of the above lemma can be founded in [19], the proof of this Lemma is not given there. Here we provide a relatively simple proof as follows.

Proof. It follows Corollary 1.6 in $[\mathbf{3}]$ that the cone $P$ is a retract of the real Banach space $E$. Assume that

$$
\begin{aligned}
& \Omega_{1}=P(\gamma, c)=\{x \in P: \gamma(x)<c\}, \\
& \Omega_{2}=P(\theta, b)=\{x \in P: \theta(x)<b\},
\end{aligned}
$$

and

$$
\Omega_{3}=P(\alpha, a)=\{x \in P: \alpha(x)<a\} .
$$


For arbitrary $x \in \overline{\Omega_{3}}$, we have $\alpha(x) \leq a$. So we further get

$$
\theta(x) \leq a<b,
$$

which implies $x \in \Omega_{2}$.

For arbitrary $x \in \overline{\Omega_{2}}$, we have $\theta(x) \leq b$. So it can further be deduced

$$
\gamma(x) \leq b<c,
$$

which indicates $x \in \Omega_{1}$.

Consequently, we have

$$
\overline{\Omega_{3}} \subset \Omega_{2} \text { and } \overline{\Omega_{2}} \subset \Omega_{1} .
$$

Note that if $x \in \Omega_{1}$, then it gives

$$
\|x\| \leq M \gamma(x) \leq M c
$$

So $\Omega_{1}, \Omega_{2}$ and $\Omega_{3}$ are bounded, nonempty, relatively open sets of $P$.

Let $u_{0}>0$ be an element of the cone $P$ and $\lambda$ be a nonnegative real number. Firstly, we claim that $x-A x \neq \lambda u_{0}$ for all $x \in \partial \Omega_{3}$. Suppose to the contrary that it is not true, then there exists $z_{3} \in \partial \Omega_{3}$ such that $z_{3}-A z_{3}=\lambda u_{0}$. In view of assumption (iii), we get

$$
a=\alpha\left(z_{3}\right)=\alpha\left(A z_{3}+\lambda u_{0}\right) \geq \alpha\left(A z_{3}\right)<a,
$$

which yields a contradiction, because Lemma 2.4 implies that $i\left(A, \Omega_{3}, P\right)=0$.

Secondly, we claim that $x-A x \neq \lambda u_{0}$ for all $x \in \partial \Omega_{1}$. If it is not true, then there exists $z_{1} \in \partial \Omega_{1}$ satisfying $z_{1}-A z_{1}=\lambda u_{0}$. From assumption (i), we have

$$
c=\gamma\left(z_{1}\right)=\gamma\left(A z_{1}+\lambda u_{0}\right) \geq \gamma\left(A z_{1}\right)<c,
$$

which is another contradiction, because Lemma 2.4 implies that $i\left(A, \Omega_{1}, P\right)=0$.

Thirdly, let $H(t, x)=t A x$. It is obvious that

$$
H:[0,1] \times \overline{\Omega_{2}} \rightarrow P,
$$

is completely continuous.

Fourthly, we claim that $H(t, x) \neq x$ for all $(t, x) \in[0,1] \times \partial \Omega_{2}$. Suppose to the contrary that it does not hold, then there exists

$$
\left(t_{2}, z_{2}\right) \in[0,1] \times \partial \Omega_{2},
$$

such that

$$
H\left(t_{2}, z_{2}\right)=z_{2}
$$

By assumption (ii), we obtain

$$
b=\theta\left(z_{2}\right)=\theta\left(t_{2} A z_{2}\right) \leq t_{2} \theta\left(A z_{2}\right)<\theta\left(A z_{2}\right)>b,
$$

apparently, which is a contradiction.

Hence, the homotopy invariance and normality properties of the fixed point index imply that

$$
i\left(A, \Omega_{2}, P\right)=i\left(0, \Omega_{2}, P\right)=1,
$$

due to that $\theta(0)=0$.

By virtue of the additivity property, we obtain

$$
i\left(A, \Omega_{2}-\overline{\Omega_{3}}, P\right)=i\left(A, \Omega_{2}, P\right)-i\left(A, \Omega_{3}, P\right)=1,
$$

and

$$
i\left(A, \Omega_{1}-\overline{\Omega_{2}}, P\right)=i\left(A, \Omega_{1}, P\right)-i\left(A, \Omega_{2}, P\right)=-1 .
$$


It follows the solution property of the fixed point index that there exists two points $x_{1}$ and $x_{2}$ for $A$ such that:

$$
x_{1} \in \Omega_{2}-\overline{\Omega_{3}} \text { which implies } a<\alpha\left(x_{1}\right) \text { with } \theta\left(x_{1}\right)<b,
$$

and

$$
x_{2} \in \Omega_{1}-\overline{\Omega_{1}} \text { which implies } b<\theta\left(x_{2}\right) \text { with } \gamma\left(x_{2}\right)<c .
$$

Consequently, we have completed the proof of Lemma 2.6.

\section{Existence Results of Problem (1.4) and (1.5)}

In this section, by using the fixed point theorems due to Avery and Henderson [3], we will discuss the existence of at least twin or arbitrary even positive solutions to problem (1.4) and (1.5) under given conditions.

Let the Banach space

$$
E=C_{l d}\left([0, \sigma(T)]_{\mathbb{T}} \rightarrow \mathbb{R}\right),
$$

with the norm

$$
\|u\|=\max \left\{\sup _{t \in[0, T]_{\mathbb{T}}}|u(t)|, \sup _{t \in[0, T]_{\mathbb{T}}}\left|u^{\Delta}(t)\right|\right\},
$$

and we define the cone $P \subset E$ by

$$
P=\left\{\begin{array}{c}
u \in E: u(t) \geq 0 \text { for } t \in[0, \sigma(T)]_{\mathbb{T}} \text { and } \\
u^{\Delta \nabla}(t) \leq 0, u^{\Delta}(t) \geq 0 \text { for } t \in[0, T]_{\mathbb{T}}, u^{\Delta}(T)=0
\end{array}\right\} .
$$

In order to obtain the main results, we need to use the following useful lemmas.

LEMMA 3.1. If $u \in P$, then we have

(i): $u(t) \geq \frac{t}{T} u(T)=\frac{t}{T} \sup _{t \in[0, T]_{\mathbb{T}}} u(t)$ for $t \in[0, T]_{\mathbb{T}}$;

(ii): $s u(t) \leq t u(s)$ for $s, t \in[0, T]_{\mathbb{T}}$ and $s \leq t$.

Proof. The proof can be made by using a similar way to that of Lemma 3.1 in [24], so we omit it.

Lemma 3.2. For any $u \in P$, there exists a real number $M^{*}>0$ such that

$$
\sup _{t \in[0, T]_{\mathbb{T}}} u(t) \leq M^{*} \sup _{t \in[0, T]_{\mathbb{T}}} u^{\Delta}(t)=M^{*} u^{\Delta}(0),
$$

where $M^{*}=\max \left\{1, B \sum_{i=1}^{m-2} a_{i}+T\right\}$.

Since $u(t)=u(0)+\int_{0}^{t} u^{\Delta}(t) \Delta t \quad$ and $u^{\Delta}(t) \geq u^{\Delta}(T)=0$, the proof of Lemma 3.2 can be directly seen by

$$
\begin{aligned}
u(T) & =\sup _{t \in[0, T]_{\mathbb{T}}} u(t), \\
& \leq \sup _{t \in[0, T]_{\mathbb{T}}}\left\{B_{0}\left(\sum_{i=1}^{m-2} a_{i} u^{\Delta}\left(\xi_{i}\right)\right)+\int_{0}^{t} u^{\Delta}(t) \Delta t\right\}, \\
& \leq\left(B \sum_{i=1}^{m-2} a_{i}+T\right) \sup _{t \in[0, T]_{\mathbb{T}}} u^{\Delta}(t) .
\end{aligned}
$$


Lemma 3.2 implies that $\|u\| \leq M^{*} u^{\Delta}(0)$ for $u \in P$. In view of (1.4) and (1.5), it is easy to see that

$$
\begin{aligned}
u(t)= & B_{0}\left(\sum_{i=1}^{m-2} a_{i} \varphi_{q}\left(\int_{\xi_{i}}^{T} h(s) f\left(s, u(s), u^{\Delta}(s)\right) \nabla s\right)\right) \\
& +\int_{0}^{t} \varphi_{q}\left(\int_{\tau}^{T} h(s) f\left(s, u(s), u^{\Delta}(s)\right) \nabla s\right) \Delta \tau .
\end{aligned}
$$

Define the operator $A: P \rightarrow E$ as

$$
\begin{aligned}
A u(t)= & B_{0}\left(\sum_{i=1}^{m-2} a_{i} \varphi_{q}\left(\int_{\xi_{i}}^{T} h(s) f\left(s, u(s), u^{\Delta}(s)\right) \nabla s\right)\right) \\
& +\int_{0}^{t} \varphi_{q}\left(\int_{\tau}^{T} h(s) f\left(s, u(s), u^{\Delta}(s)\right) \nabla s\right) \Delta \tau .
\end{aligned}
$$

It is apparent that $\varphi_{p}(u)$ is not differentiable at $u=0$ for $1<p<2$, as a result of that the chain rule can not be used to obtain that $A u \in P$ for $u \in P$. So we still need to prove the following Lemma:

Lemma 3.3. A: $P \rightarrow P$ is completely continuous.

Proof. Firstly, we show that $A: P \rightarrow P$.

From (S1), it is obvious that

$$
(A u)(t) \geq 0 \text { for } t \in[0, T]_{\mathbb{T}} \subset[0, \sigma(T)]_{\mathbb{T}},
$$

and

In addition,

$$
(A u)^{\Delta}(T)=0
$$

$$
(A u)^{\Delta}(t)=\varphi_{q}\left(\int_{t}^{T} h(r) f\left(s, u, u^{\Delta}\right) \nabla s\right) \geq 0,
$$

is continuous and nonincreasing in $[0, T]_{\mathbb{T}}$, and it holds that

$$
\left(\int_{t}^{T} h(s) f\left(s, u, u^{\Delta}\right) \nabla s\right)^{\nabla}=-h(t) f\left(t, u, u^{\Delta}\right) \leq 0, t \in[0, T]_{\mathbb{T}} .
$$

Moreover, $\varphi_{q}(u)$ is monotone increasing and continuously differentiable function for $u>0$.

When

$$
\int_{t}^{T} h(r) f\left(s, u, u^{\Delta}\right) \nabla s>0 \text { for } t \in[0, T]_{\mathbb{T}},
$$

by the chain rule [7, Theorem 1.87$]$, we obtain

$$
(A u)^{\Delta \nabla}(t) \leq 0 \text { for } t \in[0, T]_{\mathbb{T}} .
$$

When

$$
\int_{t}^{T} h(r) f\left(s, u, u^{\Delta}\right) \nabla s=0 \text { for } t \in[0, T]_{\mathbb{T}}
$$

we have

$$
(A u)^{\Delta \nabla}(t)=0 \text { for } t \in[0, T]_{\mathbb{T}} .
$$

Secondly, we show that $A$ maps a bounded set into a bounded set. Assume that $c>0$ is a constant and

$$
u \in \bar{P}_{c}=\left\{u \in P:\|u\|=\max \left\{\sup _{t \in[0, T]_{\mathbb{T}}}|u(t)|, \sup _{t \in[0, T]_{\mathbb{T}}}\left|u^{\Delta}(t)\right|\right\} \leq c\right\} .
$$


Note that the ld-continuity of $f$ guarantees that there is a $C>0$ such that

$$
f\left(t, u, u^{\Delta}\right) \leq \varphi_{p}(C) \text { for }\left(t, u, u^{\Delta}\right) \in[0, T]_{\mathbb{T}} \times[0, c] \times[0, c] .
$$

For $t \in[0, T]_{\mathbb{T}}$, we have

$$
\left|\varphi_{q}\left(\int_{t}^{T} h(s) f\left(s, u, u^{\Delta}\right) \nabla s\right)\right|<+\infty
$$

and

$$
\begin{aligned}
& \mid B_{0}\left(\sum_{i=1}^{m-2} a_{i} \varphi_{q}\left(\int_{\xi_{i}}^{T} h(s) f\left(s, u, u^{\Delta}\right) \nabla s\right)\right) \\
& +\int_{0}^{t} \varphi_{q}\left(\int_{\tau}^{T} h(s) f\left(s, u, u^{\Delta}\right) \nabla s\right) \Delta \tau \mid<+\infty .
\end{aligned}
$$

This implies that $A$ maps a bounded set into a bounded set.

Thirdly, for $t_{1}, t_{2} \in[0, T]_{\mathbb{T}}$, we have

$$
\begin{aligned}
& \left|(A u)\left(t_{1}\right)-(A u)\left(t_{2}\right)\right|, \\
= & \left|\int_{t_{1}}^{t_{2}} \varphi_{q}\left(\int_{\tau}^{T} h(s) f\left(s, u, u^{\Delta}\right) \nabla s\right) \Delta \tau\right|, \\
\leq & C\left|\int_{t_{1}}^{t_{2}} \varphi_{q}\left(\int_{\tau}^{T} h(s) \nabla s\right) \Delta \tau\right|, \\
\leq & C\left|t_{1}-t_{2}\right| \varphi_{q}\left(\int_{0}^{T} h(s) \nabla s\right) \rightarrow 0 \text { as } t_{1} \rightarrow t_{2} .
\end{aligned}
$$

The Arzela-Ascoli Theorem on time scales tells us that $A \bar{P}_{c}$ is relatively compact $[\mathbf{1}]$.

We now claim that $A$ : $\bar{P}_{c} \rightarrow P$ is continuous. Assume that $\left\{u_{n}\right\}_{n=1}^{\infty} \subset \bar{P}_{c}$ and $\lim _{n \rightarrow \infty}\left\|u_{n}-u_{0}\right\| \rightarrow 0$. This implies that

$$
\lim _{n \rightarrow \infty}\left|u_{n}-u_{0}\right| \rightarrow 0 \text { and } \lim _{n \rightarrow \infty}\left|u_{n}^{\Delta}-u_{0}^{\Delta}\right| \rightarrow 0 .
$$

Since $\left\{\left(A u_{n}\right)(t)\right\}_{n=1}^{\infty}$ is uniformly bounded and equicontinuous on $[0, T]_{\mathbb{T}}$, there exists a uniformly convergent subsequence in $\left\{\left(A u_{n}\right)(t)\right\}_{n=1}^{\infty}$. Let $\left\{\left(A u_{n(m)}\right)(t)\right\}_{m=1}^{\infty}$ be a subsequence which converges to $v(t)$ uniformly on $[0, T]_{\mathbb{T}}$. Observe that

$$
\begin{aligned}
& \left(A u_{n}\right)(t)=B_{0}\left(\sum_{i=1}^{m-2} a_{i} \varphi_{q}\left(\int_{\xi_{i}}^{T} h(s) f\left(s, u_{n}, u_{n}^{\Delta}\right) \nabla s\right)\right) \\
& +\int_{0}^{t} \varphi_{q}\left(\int_{\tau}^{T} h(s) f\left(s, u_{n}, u_{n}^{\Delta}\right) \nabla s\right) \Delta \tau .
\end{aligned}
$$

By using (3.1) and (3.2), inserting $u_{n(m)}$ into the above and then letting $m \rightarrow \infty$, we obtain

$$
\begin{aligned}
& v(t)=B_{0}\left(\sum_{i=1}^{m-2} a_{i} \varphi_{q}\left(\int_{\xi_{i}}^{T} h(s) f\left(s, u_{0}, u_{0}^{\Delta}\right) \nabla s\right)\right) \\
& +\int_{0}^{t} \varphi_{q}\left(\int_{\tau}^{T} h(s) f\left(s, u_{0}, u_{0}^{\Delta}\right) \nabla s\right) \Delta \tau .
\end{aligned}
$$

Here we used the Lebesgue's dominated convergence Theorem on time scales [2]. From the definition of $A$, we know that $v(t)=A u_{0}(t)$ on $[0, T]_{\mathbb{T}}$. This shows that each subsequence of $\left\{A u_{n}(t)\right\}_{n=1}^{\infty}$ uniformly converges to $\left(A u_{0}\right)(t)$. So the sequence $\left\{\left(A u_{n}\right)(t)\right\}_{n=1}^{\infty}$ uniformly converges to $\left(A u_{0}\right)(t)$, which implies that $A$ is continuous at $u_{0} \in \bar{P}_{c}$. That is, $A$ is continuous on $\bar{P}_{c}$ since $u_{0}$ is arbitrary. Thus, $A$ is completely continuous. The proof is completed. 
It is easy to see that each fixed point of $A$ is a solution of the problem (1.4) and (1.5). For $u \in P$, we define the nonnegative, increasing, continuous functionals $\gamma, \theta$ and $\alpha$ by

$$
\begin{aligned}
& \gamma(u)=\varepsilon \max _{t \in[0, T]_{\mathbb{T}}} u^{\Delta}(t)+\min _{t \in[\eta, T]_{\mathbb{T}}} u(t)=\varepsilon u^{\Delta}(0)+u(\eta), \\
& \theta(u)=\varepsilon \max _{t \in[0, T]_{\mathbb{T}}} u^{\Delta}(t)+\max _{t \in[0, \eta]_{\mathbb{T}}} u(t)=\varepsilon u^{\Delta}(0)+u(\eta),
\end{aligned}
$$

and

$$
\alpha(u)=\varepsilon \max _{t \in[0, T]_{\mathbb{T}}} u^{\Delta}(t)+\max _{t \in[0, r]_{\mathbb{T}}} u(t)=\varepsilon u^{\Delta}(0)+u(r) .
$$

where $\varepsilon$ is an arbitrary positive number.

It is obvious that

$$
\gamma(u) \leq \theta(u) \leq \alpha(u) \text { for each } u \in P .
$$

By Lemma 3.2, one can obtain

$$
\|u\| \leq M^{*} u^{\Delta}(0)<\frac{M^{*}}{\varepsilon} \varepsilon u^{\Delta}(0)+\frac{M^{*}}{\varepsilon} u(\eta)=\frac{M^{*}}{\varepsilon} \gamma(u) \text { for all } u \in P .
$$

In addition, for the positive constant $b^{\prime}$, we have

$$
\theta(\lambda u)=\lambda \theta(u), \quad 0 \leq \lambda \leq 1 \text { and } u \in \partial P\left(\theta, b^{\prime}\right) .
$$

For the notational convenience, we denote

$$
\begin{gathered}
M=\left(A^{\prime} \sum_{i=1}^{m-2} a_{i}+\eta\right) \varphi_{q}\left(\int_{\eta}^{T} h(s) \nabla s\right), \\
N=\left(1+B \sum_{i=1}^{m-2} a_{i}+\eta\right) \varphi_{q}\left(\int_{0}^{T} h(s) \nabla s\right),
\end{gathered}
$$

and

$$
L=\left(1+A^{\prime} \sum_{i=1}^{m-2} a_{i}+r\right) \varphi_{q}\left(\int_{r}^{T} h(s) \nabla s\right) .
$$

We are now ready to present our main result in this section:

THEOREM 3.4. Suppose that there are three positive numbers $a^{\prime}, b^{\prime}$ and $c^{\prime}$ such that $0<a^{\prime}<\frac{L b^{\prime}}{N}<\frac{c^{\prime} \eta L}{T N}$ holds. Suppose that $\varepsilon$ is an arbitrary small positive number and $f\left(t, u, u^{\Delta}\right)$ satisfies the following three conditions:

(i) $f\left(t, u, u^{\Delta}\right)>\varphi_{p}\left(\frac{c^{\prime}}{M}\right)$ for $\left(t, u, u^{\Delta}\right) \in[\eta, T]_{\mathbb{T}} \times\left[c^{\prime}-\varepsilon, \frac{T}{\eta} c^{\prime}\right] \times\left[0, \frac{c^{\prime}}{\varepsilon}\right]$;

(ii) $f\left(t, u, u^{\Delta}\right)<\varphi_{p}\left(\frac{b^{\prime}}{N}\right)$ for $\left(t, u, u^{\Delta}\right) \in[0, T]_{\mathbb{T}} \times\left[0, \frac{T}{\eta} b^{\prime}\right] \times\left[0, \frac{b^{\prime}}{\varepsilon}\right]$;

(iii) $f\left(t, u, u^{\Delta}\right)>\varphi_{p}\left(\frac{a^{\prime}}{L}\right)$ for $\left(t, u, u^{\Delta}\right) \in[r, T]_{\mathbb{T}} \times\left[0, \frac{T}{r} a^{\prime}\right] \times\left[0, \frac{a^{\prime}}{\varepsilon}\right]$.

Then problem (1.4) and (1.5) has at least two positive solutions $u_{1}$ and $u_{2}$ such that

$$
a^{\prime}<\varepsilon \max _{t \in[0, T]_{\mathbb{T}}} u_{1}^{\Delta}(t)+\max _{t \in[0, r]_{\mathbb{T}}} u_{1}(t) \text { with } \varepsilon \max _{t \in[0, T]_{\mathbb{T}}} u_{1}^{\Delta}(t)+\max _{t \in[0, \eta]_{\mathbb{T}}} u_{1}(t)<b^{\prime}
$$

and

(3.4) $b^{\prime}<\varepsilon \max _{t \in[0, T]_{\mathbb{T}}} u_{2}^{\Delta}(t)+\max _{t \in[0, \eta]_{\mathbb{T}}} u_{2}(t)$ with $\varepsilon \max _{t \in[0, T]_{\mathbb{T}}} u_{2}^{\Delta}(t)+\min _{t \in[\eta, T]_{\mathbb{T}}} u_{2}^{\Delta}(t)<c^{\prime}$. 
Proof. By the definition of the completely continuous operator $A$ and its properties, it suffices to show that all conditions of Lemma 2.5 hold with respect to $A$.

In the first step, we verify that if $u \in \partial P\left(\gamma, c^{\prime}\right)$, then $\gamma(A u)>c^{\prime}$.

Suppose that $u \in \partial P\left(\gamma, c^{\prime}\right)$, then we have

$$
\gamma(u)=\varepsilon \max _{t \in[0, T]_{\mathbb{T}}} u^{\Delta}(t)+\min _{t \in[\eta, T]_{\mathbb{T}}} u(t)=\varepsilon u^{\Delta}(0)+u(\eta)=c^{\prime} .
$$

Since

$$
u^{\Delta}(t) \geq 0 \text { and } u(t) \geq 0 \text { for } t \in[0, T]_{\mathbb{T}},
$$

we have

$$
0=u^{\Delta}(T) \leq u^{\Delta}(t) \leq u^{\Delta}(0) \leq \frac{1}{\varepsilon} \varepsilon u^{\Delta}(0)+\frac{1}{\varepsilon} u(\eta) \leq \frac{1}{\varepsilon} \gamma(u)=\frac{c^{\prime}}{\varepsilon} \text { for } t \in[0, T]_{\mathbb{T}} .
$$

Lemma 3.1 implies that

$$
\max _{t \in[0, T]_{\mathbb{T}}} u(t) \leq \frac{T}{\eta} u(\eta) \leq \frac{T}{\eta} c^{\prime}
$$

and by (3.5) we have

$$
c^{\prime}-\varepsilon \leq u(t) \leq \frac{T}{\eta} c^{\prime}, \quad t \in[\eta, T]_{\mathbb{T}} .
$$

By virtue of condition (i) of Theorem 3.4 and (1.7), we deduce that

$$
\begin{aligned}
\gamma(A u)= & \varepsilon(A u)^{\Delta}(0)+A u(\eta) \\
= & \varepsilon \varphi_{q}\left(\int_{0}^{T} h(s) f\left(s, u(s), u^{\Delta}(s)\right) \nabla s\right), \\
& +B_{0}\left(\sum_{i=1}^{m-2} a_{i} \varphi_{q}\left(\int_{\xi_{i}}^{T} h(s) f\left(s, u(s), u^{\Delta}(s)\right) \nabla s\right)\right) \\
& +\int_{0}^{\eta} \varphi_{q}\left(\int_{\tau}^{T} h(s) f\left(s, u(s), u^{\Delta}(s)\right) \nabla s\right) \Delta \tau, \\
> & A^{\prime} \sum_{i=1}^{m-2} a_{i} \varphi_{q}\left(\int_{0}^{T} h(s) f\left(s, u(s), u^{\Delta}(s)\right) \nabla s\right) \\
& +\int_{0}^{\eta} \varphi_{q}\left(\int_{\tau}^{T} h(s) f\left(s, u(s), u^{\Delta}(s)\right) \nabla s\right) \Delta \tau, \\
\geq & \left(A^{\prime} \sum_{i=1}^{m-2} a_{i}+\eta\right) \varphi_{q}\left(\int_{\eta}^{T} h(s) f\left(s, u(s), u^{\Delta}(s)\right) \nabla s\right), \\
> & \left(A^{\prime} \sum_{i=1}^{m-2} a_{i}+\eta\right) \varphi_{q}\left(\int_{\eta}^{T} h(s) \varphi_{p}\left(\frac{c^{\prime}}{M}\right) \nabla s\right), \\
= & \frac{c^{\prime}}{M}\left(A^{\prime} \sum_{i=1}^{m-2} a_{i}+\eta\right) \varphi_{q}\left(\int_{\eta}^{T} h(s) \nabla s\right), \\
= & c^{\prime} .
\end{aligned}
$$

In the second step, we claim that $\theta(A u)<b^{\prime}$ for $u \in \partial P\left(\theta, b^{\prime}\right)$. 
If we choose $u \in \partial P\left(\theta, b^{\prime}\right)$, then we have

$$
\theta(u)=\varepsilon \max _{t \in[0, T]_{\mathbb{T}}} u^{\Delta}(t)+\max _{t \in[0, \eta]_{\mathbb{T}}} u(t)=\varepsilon u^{\Delta}(0)+u(\eta)=b^{\prime},
$$

which implies

$$
\max _{t \in[0, \eta]_{\mathbb{T}}} u(t)=u(\eta) \leq b^{\prime} \text { and } \varepsilon \max _{t \in[0, T]_{\mathbb{T}}} u^{\Delta}(t) \leq b^{\prime}
$$

so we have

$$
0 \leq u^{\Delta}(t) \leq \frac{b^{\prime}}{\varepsilon} \text { for } t \in[0, T]_{\mathbb{T}} .
$$

In view of Lemma 3.1, we have

$$
\max _{t \in[0, T]_{\mathbb{T}}} u(t) \leq \frac{T}{\eta} u(\eta) \leq \frac{T}{\eta} b^{\prime} .
$$

Consequently, we derive that

$$
0 \leq u(t) \leq \frac{T}{\eta} b^{\prime}, \quad t \in[0, T]_{\mathbb{T}} .
$$

Using assumption (ii) in Theorem 3.4 and (1.7), we obtain

$$
\begin{aligned}
& \theta(A u)=(A u)^{\Delta}(0)+(A u)(\eta), \\
& =\varphi_{q}\left(\int_{0}^{T} h(s) f\left(s, u(s), u^{\Delta}(s)\right) \nabla s\right) \\
& +B_{0}\left(\sum_{i=1}^{m-2} a_{i} \varphi_{q}\left(\int_{\xi_{i}}^{T} h(s) f\left(s, u(s), u^{\Delta}(s)\right) \nabla s\right)\right) \\
& +\int_{0}^{\eta} \varphi_{q}\left(\int_{\tau}^{T} h(s) f\left(s, u(s), u^{\Delta}(s)\right) \nabla s\right) \Delta \tau \\
& <\varphi_{q}\left(\int_{0}^{T} h(s) f\left(s, u(s), u^{\Delta}(s)\right) \nabla s\right) \\
& +B \sum_{i=1}^{m-2} a_{i} \varphi_{q}\left(\int_{\xi_{i}}^{T} h(s) f\left(s, u(s), u^{\Delta}(s)\right) \nabla s\right) \\
& +\eta \varphi_{q}\left(\int_{0}^{T} h(s) f\left(s, u(s), u^{\Delta}(s)\right) \nabla s\right), \\
& <\left(1+B \sum_{i=1}^{m-2} a_{i}+\eta\right) \varphi_{q}\left(\int_{0}^{T} h(s) \varphi_{p}\left(\frac{b^{\prime}}{N}\right) \nabla s\right), \\
& =\frac{b^{\prime}}{N}\left(1+B \sum_{i=1}^{m-2} a_{i}+\eta\right) \varphi_{q}\left(\int_{0}^{T} h(s) \nabla s\right), \\
& =b^{\prime} \text {. }
\end{aligned}
$$

In the third step, we prove that

$$
P\left(\alpha, a^{\prime}\right) \neq \emptyset
$$

and

$$
\alpha(A u)>a^{\prime} \text { for all } u \in \partial P\left(\alpha, a^{\prime}\right)
$$


In fact, the constant function $\frac{a^{\prime}}{2} \in P\left(\alpha, a^{\prime}\right)$, for $u \in \partial P\left(\alpha, a^{\prime}\right)$, we have

$$
\alpha(u)=\varepsilon \max _{t \in[0, T]_{\mathbb{T}}} u^{\Delta}(t)+\max _{t \in[0, r]_{\mathbb{T}}} u(t)=\varepsilon u^{\Delta}(0)+u(r)=a^{\prime},
$$

which implies

$$
0 \leq u(t) \leq a^{\prime} \text { for } t \in[0, r]_{\mathbb{T}} \text { and } 0 \leq u^{\Delta}(t) \leq \frac{a^{\prime}}{\varepsilon} \text { for } t \in[0, T]_{\mathbb{T}} .
$$

In view of Lemma 3.1 , we have

$$
\max _{t \in[0, T]_{\mathbb{T}}} u(t) \leq \frac{T}{r} u(r)=\frac{T}{r} r^{\prime} .
$$

That is,

$$
0 \leq u(t) \leq \frac{T}{r} a^{\prime}, \quad t \in[r, T]_{\mathbb{T}}
$$

Using assumption (iii) in Theorem 3.4 and (1.7), we have

$$
\begin{aligned}
\alpha(A u)= & (A u)^{\Delta}(0)+(A u)(\eta), \\
= & \varphi_{q}\left(\int_{0}^{T} h(s) f\left(s, u(s), u^{\Delta}(s)\right) \nabla s\right), \\
& +B_{0}\left(\sum_{i=1}^{m-2} a_{i} \varphi_{q}\left(\int_{\xi_{i}}^{T} h(s) f\left(s, u(s), u^{\Delta}(s)\right) \nabla s\right)\right) \\
& +\int_{0}^{r} \varphi_{q}\left(\int_{\tau}^{T} h(s) f\left(s, u(s), u^{\Delta}(s)\right) \nabla s\right) \Delta \tau, \\
> & \varphi_{q}\left(\int_{0}^{T} h(s) f\left(s, u(s), u^{\Delta}(s)\right) \nabla s\right) \\
& +A^{\prime} \sum_{i=1}^{m-2} a_{i} \varphi_{q}\left(\int_{r}^{T} h(s) f\left(s, u(s), u^{\Delta}(s)\right) \nabla s\right) \\
& +\int_{0}^{r} \varphi_{q}\left(\int_{r}^{T} h(s) f\left(s, u(s), u^{\Delta}(s)\right) \nabla s\right) \Delta \tau, \\
> & \left(1+A^{\prime} \sum_{i=1}^{m-2} a_{i}+r\right) \varphi_{q}\left(\int_{r}^{T} h(s) \varphi_{p}\left(\frac{a^{\prime}}{L}\right) \nabla s\right), \\
= & \frac{a^{\prime}}{L}\left(1+A^{\prime} \sum_{i=1}^{m-2} a_{i}+r\right) \varphi_{q}\left(\int_{r}^{T} h(s) \nabla s\right), \\
= & a^{\prime} .
\end{aligned}
$$

From the above three steps, we can see that all conditions in Lemma 2.5 are satisfied. According to Lemma 2.5, problem (1.4) and (1.5) has at least twin positive solutions $u_{1}$ and $u_{2}$, belonging to $\overline{P\left(\gamma, c^{\prime}\right)}$, and such that (3.3) and (3.4) hold. Consequently, the proof is completed.

Now we present our result on the existence of arbitrary even positive solutions of problem (1.4) and (1.5). 
TheOREM 3.5. Let $i=1,2, \ldots, n, n \in \mathbb{N}$. Suppose that there are three positive numbers $a_{i}^{\prime}, b_{i}^{\prime}$ and $c_{i}^{\prime}$ such that

$$
0<a_{1}^{\prime}<\frac{L}{N} b_{1}^{\prime}<\frac{\eta L}{T N} c_{1}^{\prime}<a_{2}^{\prime}<\frac{L}{N} b_{2}^{\prime}<\frac{\eta L}{T N} c_{2}^{\prime}<\ldots<a_{n}^{\prime}<\frac{L}{N} b_{n}^{\prime}<\frac{\eta L}{T N} c_{n}^{\prime} .
$$

When $f\left(t, u, u^{\Delta}\right)$ satisfies the following three conditions:

(i) $f\left(t, u, u^{\Delta}\right)>\varphi_{p}\left(\frac{c_{i}^{\prime}}{M}\right)$ for $\left(t, u, u^{\Delta}\right) \in[\eta, T]_{\mathbb{T}} \times\left[c_{i}^{\prime}-\varepsilon, \frac{T}{\eta} c_{i}^{\prime}\right] \times\left[0, \frac{c_{i}^{\prime}}{\varepsilon}\right]$;

(ii) $f\left(t, u, u^{\Delta}\right)<\varphi_{p}\left(\frac{b_{i}^{\prime}}{N}\right)$ for $\left(t, u, u^{\Delta}\right) \in[0, T]_{\mathbb{T}} \times\left[0, \frac{T}{\eta} b_{i}^{\prime}\right] \times\left[0, \frac{b_{i}^{\prime}}{\varepsilon}\right]$;

(iii) $f\left(t, u, u^{\Delta}\right)>\varphi_{p}\left(\frac{a_{i}^{\prime}}{L}\right)$ for $\left(t, u, u^{\Delta}\right) \in[r, T]_{\mathbb{T}} \times\left[0, \frac{T}{r} a_{i}^{\prime}\right] \times\left[0, \frac{a_{i}^{\prime}}{\varepsilon}\right]$;

then problem (1.4) and (1.5) has at least $2 n$ positive solutions.

It is noted that when $i=1$, from Theorem 3.4 it is true that problem (1.4) and (1.5) has at least twin positive solutions $u_{1}$ and $u_{2}$, belonging to $\overline{P\left(\gamma, c_{1}^{\prime}\right)}$. Using the mathematical induction, one can complete the proof of Theorem 3.5. So we omit it here.

For convenience of our statement, we denote $M^{\prime}, N^{\prime}$ and $L^{\prime}$ by

$$
\begin{gathered}
M^{\prime}=\left(1+B \sum_{i=1}^{m-2} a_{i}+\eta\right) \varphi_{q}\left(\int_{0}^{T} h(s) \nabla s\right), \\
N^{\prime}=\left(A^{\prime} \sum_{i=1}^{m-2} a_{i}+\eta\right) \varphi_{q}\left(\int_{\eta}^{T} h(s) \nabla s\right),
\end{gathered}
$$

and

$$
L^{\prime}=\left(1+B \sum_{i=1}^{m-2} a_{i}+r\right) \varphi_{q}\left(\int_{0}^{T} h(s) \nabla s\right) .
$$

In view of Lemma 2.6, by using a closely similar proof to that of Theorem 3.4, we can obtain the following result:

THEOREM 3.6. Assume that there are three positive numbers $a^{\prime}, b^{\prime}$ and $c^{\prime}$ such that $0<a^{\prime}<\frac{r}{T} b^{\prime}<\frac{r N^{\prime}}{T M^{\prime}} c^{\prime}$. Suppose that $f\left(t, u, u^{\Delta}\right)$ satisfies the following three conditions:

(i) $f\left(t, u, u^{\Delta}\right)<\varphi_{p}\left(\frac{c^{\prime}}{M^{\prime}}\right)$ for $\left(t, u, u^{\Delta}\right) \in[0, T]_{\mathbb{T}} \times\left[0, c^{\prime}\right] \times\left[0, \frac{c^{\prime}}{\varepsilon}\right]$;

(ii) $f\left(t, u, u^{\Delta}\right)>\varphi_{p}\left(\frac{b^{\prime}}{N^{\prime}}\right)$ for $\left(t, u, u^{\Delta}\right) \in[\eta, T]_{\mathbb{T}} \times\left[b^{\prime}-\varepsilon, \frac{T}{\eta} b^{\prime}\right] \times\left[0, \frac{b^{\prime}}{\varepsilon}\right]$;

(iii) $f\left(t, u, u^{\Delta}\right)<\varphi_{p}\left(\frac{a^{\prime}}{L^{\prime}}\right)$ for $\left(t, u, u^{\Delta}\right) \in[0, T]_{\mathbb{T}} \times\left[0, \frac{T}{r} a^{\prime}\right] \times\left[0, \frac{a^{\prime}}{\varepsilon}\right]$.

Then problem (1.4) and (1.5) has at least twin positive solutions $u_{1}$ and $u_{2}$ such that (3.3) and (3.4) hold.

Similarly, using the same way as that of Theorem 3.5, we can obtain the following result:

TheOREM 3.7. Let $i=1,2, \ldots, n, n \in \mathbb{N}$. Assume that there are three positive numbers $a_{i}^{\prime}, b_{i}^{\prime}$ and $c_{i}^{\prime}$ such that

$$
0<a_{1}^{\prime}<\frac{r}{T} b_{1}^{\prime}<\frac{r N^{\prime}}{T M^{\prime}} c_{1}^{\prime}<a_{2}^{\prime}<\frac{r}{T} b_{2}^{\prime}<\frac{r N^{\prime}}{T M^{\prime}} c_{2}^{\prime}<\ldots<a_{n}^{\prime}<\frac{r}{T} b_{n}^{\prime}<\frac{r N^{\prime}}{T M^{\prime}} c_{n}^{\prime} .
$$

Suppose that $f\left(t, u, u^{\Delta}\right)$ satisfies the following three conditions:

(i) $f\left(t, u, u^{\Delta}\right)<\varphi_{p}\left(\frac{c_{i}^{\prime}}{M^{\prime}}\right)$ for $\left(t, u, u^{\Delta}\right) \in[0, T]_{\mathbb{T}} \times\left[0, c_{i}^{\prime}\right] \times\left[0, \frac{c_{i}^{\prime}}{\varepsilon}\right]$;

(ii) $f\left(t, u, u^{\Delta}\right)>\varphi_{p}\left(\frac{b_{i}^{\prime}}{N^{\prime}}\right)$ for $\left(t, u, u^{\Delta}\right) \in[\eta, T]_{\mathbb{T}} \times\left[b_{i}^{\prime}-\varepsilon, \frac{T}{\eta} b_{i}^{\prime}\right] \times\left[0, \frac{b_{i}^{\prime}}{\varepsilon}\right]$;

(iii) $f\left(t, u, u^{\Delta}\right)<\varphi_{p}\left(\frac{a_{i}^{\prime}}{L^{\prime}}\right)$ for $\left(t, u, u^{\Delta}\right) \in[0, T]_{\mathbb{T}} \times\left[0, \frac{T}{r} a_{i}^{\prime}\right] \times\left[0, \frac{a_{i}^{\prime}}{\varepsilon}\right]$.

Then problem (1.4) and (1.5) has at least $2 n$ positive solutions. 


\section{Existence Results of Problem (1.4) and (1.6)}

In this section, we will apply the fixed point theorems to discuss the existence of at least twin or arbitrary even positive solutions to problem (1.4) and (1.6) under certain conditions.

Define the cone $P_{1} \subset E$ by

$$
P_{1}=\left\{\begin{array}{c}
u \in E: u(t) \geq 0 \text { for } t \in[0, \sigma(T)]_{\mathbb{T}} \text { and } \\
u^{\Delta \nabla}(t) \leq 0, u^{\Delta}(t) \leq 0 \text { for } t \in[0, T]_{\mathbb{T}}, u^{\Delta}(0)=0
\end{array}\right\} .
$$

The following two Lemmas are needed in the proofs of our main results in this section, which can be proved by using the similar arguments to that of Lemmas 3.1 and 3.2 .

LEMMA 4.1. If $u \in P_{1}$, then we have

(i): $u(t) \geq \frac{T-t}{T} \sup _{t \in[0, T]_{\mathbb{T}}} u(t)=\frac{T-t}{T} u(0)$ for $t \in[0, T]_{\mathbb{T}}$;

(ii): $(T-t) u(s) \leq(T-s) u(t)$ for $s, t \in[0, T]_{\mathbb{T}}$ and $s \leq t$.

LEMMA 4.2. For any $u \in P_{1}$, there exists a real number $M_{1}^{*}>0$ such that

$$
\sup _{t \in[0, T]_{\mathbb{T}}} u(t) \leq M_{1}^{*} \sup _{t \in[0, T]_{\mathbb{T}}} u^{\Delta}(t)=M_{1}^{*}\left|u^{\Delta}(T)\right|
$$

where $M_{1}^{*}=\max \left\{1, B \sum_{i=1}^{m-2} b_{i}+T\right\}$.

Clearly, one can see that

$$
\|u\| \leq M_{1}^{*}\left|u^{\Delta}(T)\right| \text { for } u \in P_{1} .
$$

To discuss the existence of positive solutions to problem (1.4) and (1.6), we define a new operator $A_{1}: P_{1} \rightarrow E$ by

$$
\begin{aligned}
A_{1} u(t)= & B_{1}\left(\sum_{i=1}^{m-2} b_{i} \varphi_{q}\left(\int_{0}^{\xi_{i}^{\prime}} h(s) f\left(s, u(s), u^{\Delta}(s)\right) \nabla s\right)\right) \\
& +\int_{t}^{T} \varphi_{q}\left(\int_{0}^{\tau} h(s) f\left(s, u(s), u^{\Delta}(s)\right) \nabla s\right) \Delta \tau .
\end{aligned}
$$

However, using the same arguments as that in the preceding section, it is not difficult to obtain that $A_{1}: P_{1} \rightarrow P_{1}$ is completely continuous and each fixed point of $A$ is a solution of the problem (1.4) and (1.6).

For $u \in P_{1}$, we define the nonnegative, increasing, continuous functionals $\gamma_{1}$, $\theta_{1}$ and $\alpha_{1}$ by

and

$$
\begin{aligned}
& \gamma_{1}(u)=\varepsilon\left|\max _{t \in[0, T]_{\mathbb{T}}} u^{\Delta}(t)\right|+\min _{t \in[0, \xi]_{\mathbb{T}}} u(t)=\varepsilon\left|u^{\Delta}(T)\right|+u(\xi), \\
& \theta_{1}(u)=\varepsilon\left|\max _{t \in[0, T]_{\mathbb{T}}} u^{\Delta}(t)\right|+\max _{t \in[\xi, T]_{\mathbb{T}}} u(t)=\varepsilon\left|u^{\Delta}(T)\right|+u(\xi),
\end{aligned}
$$

It is obvious that

$$
\alpha_{1}(u)=\varepsilon\left|\max _{t \in[0, T]_{\mathbb{T}}} u^{\Delta}(t)\right|+\max _{t \in[l, T]_{\mathbb{T}}} u(t)=\varepsilon\left|u^{\Delta}(T)\right|+u(l) .
$$

$$
\gamma_{1}(u) \leq \theta_{1}(u) \leq \alpha_{1}(u) \text { for each } u \in P_{1} .
$$

In terms of Lemma 3.2, one has

$$
\|u\| \leq M_{1}^{*}\left|u^{\Delta}(T)\right|=\frac{M_{1}^{*}}{\varepsilon} \varepsilon\left|u^{\Delta}(T)\right|<\frac{M_{1}^{*}}{\varepsilon} \gamma_{1}(u) \text { for all } u \in P_{1} .
$$


In addition, we note that

$$
\theta_{1}(\lambda u)=\lambda \theta_{1}(u), 0 \leq \lambda \leq 1 \text { and } u \in \partial P\left(\theta, b^{\prime}\right) .
$$

For the notational convenience, we denote

$$
\begin{gathered}
M_{1}=\left(A^{\prime} \sum_{i=1}^{m-2} b_{i}+T-\xi\right) \varphi_{q}\left(\int_{0}^{\xi} h(s) \nabla s\right), \\
N_{1}=\left(1+B \sum_{i=1}^{m-2} b_{i}+T-\xi\right) \varphi_{q}\left(\int_{0}^{T} h(s) \nabla s\right),
\end{gathered}
$$

and

$$
L_{1}=\left(1+A^{\prime} \sum_{i=1}^{m-2} b_{i}+T-l\right) \varphi_{q}\left(\int_{0}^{l} h(s) \nabla s\right) .
$$

In terms of operator $A_{1}$, which essentially has the same reasoning as the Theorems and Lemmas for problem (1.4) and (1.5) in the preceding section, we have the following results:

THEOREM 4.3. Suppose that there are three positive numbers $a^{\prime}, b^{\prime}$ and $c^{\prime}$ such that

$$
0<a^{\prime}<\frac{L_{1}}{N_{1}} b^{\prime}<\frac{(T-\xi) L_{1}}{T N_{1}} c^{\prime},
$$

and $f\left(t, u, u^{\Delta}\right)$ satisfies the following three conditions:

(i) $f\left(t, u, u^{\Delta}\right)>\varphi_{p}\left(\frac{c^{\prime}}{M_{1}}\right)$ for $\left(t, u, u^{\Delta}\right) \in[0, \xi]_{\mathbb{T}} \times\left[c^{\prime}-\varepsilon, \frac{T}{T-\xi} c^{\prime}\right] \times\left[0, \frac{c^{\prime}}{\varepsilon}\right]$;

(ii) $f\left(t, u, u^{\Delta}\right)<\varphi_{p}\left(\frac{b^{\prime}}{N_{1}}\right)$ for $\left(t, u, u^{\Delta}\right) \in[0, T]_{\mathbb{T}} \times\left[0, \frac{T}{T-\xi} b^{\prime}\right] \times\left[0, \frac{b^{\prime}}{\varepsilon}\right]$;

(iii) $f\left(t, u, u^{\Delta}\right)>\varphi_{p}\left(\frac{a^{\prime}}{L_{1}}\right)$ for $\left(t, u, u^{\Delta}\right) \in[l, T]_{\mathbb{T}} \times\left[0, \frac{T}{T-l} a^{\prime}\right] \times\left[0, \frac{a^{\prime}}{\varepsilon}\right]$.

Then problem (1.4) and (1.6) has at least twin positive solutions $u_{1}$ and $u_{2}$ such that

$$
a^{\prime}<\varepsilon\left|\max _{t \in[0, T]_{\mathbb{T}}} u_{1}^{\Delta}(t)\right|+\max _{t \in[l, T]_{\mathbb{T}}} u_{1}(t) \text { with } \varepsilon\left|\max _{t \in[0, T]_{\mathbb{T}}} u_{1}^{\Delta}(t)\right|+\max _{t \in[\xi, T]_{\mathbb{T}}} u_{1}(t)<b^{\prime},
$$

and

$$
b^{\prime}<\varepsilon\left|\max _{t \in[0, T]_{\mathbb{T}}} u_{2}^{\Delta}(t)\right|+\max _{t \in[\xi, T]_{\mathbb{T}}} u_{2}(t) \text { with } \varepsilon\left|\max _{t \in[0, T]_{\mathbb{T}}} u_{2}^{\Delta}(t)\right|+\min _{t \in[0, \xi]_{\mathbb{T}}} u_{2}(t)<c^{\prime} .
$$

TheOREM 4.4. Let $i=1,2, \ldots, n, n \in \mathbb{N}$. Suppose that there are three positive numbers $a_{i}^{\prime}, b_{i}^{\prime}$ and $c_{i}^{\prime}$ such that

$$
\begin{aligned}
0 & <a_{1}^{\prime}<\frac{L_{1}}{N_{1}} b_{1}^{\prime}<\frac{(T-\xi) L_{1}}{T N_{1}} c_{1}^{\prime}<a_{2}^{\prime}<\frac{L_{1}}{N_{1}} b_{2}^{\prime}<\frac{(T-\xi) L_{1}}{T N_{1}} c_{2}^{\prime} \\
& <\ldots<a_{n}^{\prime}<\frac{L_{1}}{N_{1}} b_{n}^{\prime}<\frac{(T-\xi) L_{1}}{T N_{1}} c_{n}^{\prime}
\end{aligned}
$$

and $f\left(t, u, u^{\Delta}\right)$ satisfies the following three conditions:

(i) $f\left(t, u, u^{\Delta}\right)>\varphi_{p}\left(\frac{c_{i}^{\prime}}{M_{1}}\right)$ for $\left(t, u, u^{\Delta}\right) \in[0, \xi]_{\mathbb{T}} \times\left[c_{i}^{\prime}-\varepsilon, \frac{T}{T-\xi} c_{i}^{\prime}\right] \times\left[0, \frac{c_{i}^{\prime}}{\varepsilon}\right]$;

(ii) $f\left(t, u, u^{\Delta}\right)<\varphi_{p}\left(\frac{b_{i}^{\prime}}{N_{1}}\right)$ for $\left(t, u, u^{\Delta}\right) \in[0, T]_{\mathbb{T}} \times\left[0, \frac{T}{T-\xi} b_{i}^{\prime}\right] \times\left[0, \frac{b_{i}^{\prime}}{\varepsilon}\right]$;

(iii) $f\left(t, u, u^{\Delta}\right)>\varphi_{p}\left(\frac{a_{i}^{\prime}}{L_{1}}\right)$ for $\left(t, u, u^{\Delta}\right) \in[l, T]_{\mathbb{T}} \times\left[0, \frac{T}{T-l} a_{i}^{\prime}\right] \times\left[0, \frac{a_{i}^{\prime}}{\varepsilon}\right]$.

Then problem (1.4) and (1.6) has at least $2 n$ positive solutions. 
In view of Lemma 2.6, if we denote $M^{\prime}, N^{\prime}$ and $L^{\prime}$ by

$$
\begin{gathered}
M_{1}^{\prime}=\left(1+B \sum_{i=1}^{m-2} b_{i}+T-\xi\right) \varphi_{q}\left(\int_{0}^{T} h(s) \nabla s\right), \\
N_{1}^{\prime}=\left(A^{\prime} \sum_{i=1}^{m-2} b_{i}+T-\xi\right) \varphi_{q}\left(\int_{\xi}^{T} h(s) \nabla s\right)
\end{gathered}
$$

and

$$
L_{1}^{\prime}=\left(1+B \sum_{i=1}^{m-2} b_{i}+T-l\right) \varphi_{q}\left(\int_{0}^{T} h(s) \nabla s\right),
$$

then we have the following results for the problem (1.4) and (1.6):

THEOREM 4.5. Assume that there are three positive numbers $a^{\prime}, b^{\prime}$ and $c^{\prime}$ such that

$$
0<a^{\prime}<\frac{T-L_{1}^{\prime}}{T} b^{\prime}<\frac{\left(T-L_{1}^{\prime}\right) N_{1}^{\prime}}{T M_{1}^{\prime}} c^{\prime} .
$$

Suppose that $f\left(t, u, u^{\Delta}\right)$ satisfies the following three conditions:

(i) $f\left(t, u, u^{\Delta}\right)<\varphi_{p}\left(\frac{c^{\prime}}{M_{1}^{\prime}}\right)$ for $\left(t, u, u^{\Delta}\right) \in[0, T]_{\mathbb{T}} \times\left[0, \frac{T}{T-\xi} c^{\prime}\right] \times\left[0, \frac{c^{\prime}}{\varepsilon}\right]$;

(ii) $f\left(t, u, u^{\Delta}\right)>\varphi_{p}\left(\frac{b}{N_{1}^{\prime}}\right)$ for $\left(t, u, u^{\Delta}\right) \in[\xi, T]_{\mathbb{T}} \times\left[b^{\prime}-\varepsilon, \frac{T}{T-\xi} b^{\prime}\right] \times\left[0, \frac{b^{\prime}}{\varepsilon}\right]$;

(iii) $f\left(t, u, u^{\Delta}\right)<\varphi_{p}\left(\frac{a^{\prime}}{L_{1}^{\prime}}\right)\left(t, u, u^{\Delta}\right) \in[0, T]_{\mathbb{T}} \times\left[0, \frac{T}{T-l} a^{\prime}\right] \times\left[0, \frac{a^{\prime}}{\varepsilon}\right]$.

Then problem (1.4) and (1.6) has at least twin positive solutions $u_{1}$ and $u_{2}$ such that

$$
a^{\prime}<\varepsilon\left|\max _{t \in[0, T]_{\mathbb{T}}} u_{1}^{\Delta}(t)\right|+\max _{t \in[l, T]_{\mathbb{T}}} u_{1}(t) \text { with } \varepsilon\left|\max _{t \in[0, T]_{\mathbb{T}}} u_{1}^{\Delta}(t)\right|+\max _{t \in[\xi, T]_{\mathbb{T}}} u_{1}(t)<b^{\prime},
$$

and

$$
b^{\prime}<\varepsilon\left|\max _{t \in[0, T]_{\mathbb{T}}} u_{2}^{\Delta}(t)\right|+\max _{t \in[\xi, T]_{\mathbb{T}}} u_{2}(t) \text { with } \varepsilon\left|\max _{t \in[0, T]_{\mathbb{T}}} u_{2}^{\Delta}(t)\right|+\min _{t \in[0, \xi]_{\mathbb{T}}} u_{2}(t)<c^{\prime} .
$$

TheOREm 4.6. Let $i=1,2, \ldots, n, n \in \mathbb{N}$. Assume that there are three positive numbers $a_{i}^{\prime}, b_{i}^{\prime}$ and $c_{i}^{\prime}$ such that

$$
\begin{aligned}
a_{1}^{\prime} & <\frac{T-L_{1}^{\prime}}{T} b_{1}^{\prime}<\frac{\left(T-L_{1}^{\prime}\right) N_{1}^{\prime} c_{1}^{\prime}}{T M_{1}^{\prime}}<a_{2}^{\prime}<\frac{T-L_{1}^{\prime}}{T} b_{2}^{\prime}<\frac{\left(T-L_{1}^{\prime}\right) N_{1}^{\prime} c_{2}^{\prime}}{T M_{1}^{\prime}} \\
& <\ldots<a_{n}^{\prime}<\frac{T-L_{1}^{\prime}}{T} b_{n}^{\prime}<\frac{\left(T-L_{1}^{\prime}\right) N_{1}^{\prime} c_{n}^{\prime}}{T M_{1}^{\prime}} .
\end{aligned}
$$

Suppose that $f\left(t, u, u^{\Delta}\right)$ satisfies the following three conditions:

(i) $f\left(t, u, u^{\Delta}\right)<\varphi_{p}\left(\frac{c_{i}^{\prime}}{M_{1}^{\prime}}\right)$ for $\left(t, u, u^{\Delta}\right) \in[0, T]_{\mathbb{T}} \times\left[0, \frac{T}{T-\xi} c_{i}^{\prime}\right] \times\left[0, \frac{c_{i}^{\prime}}{\varepsilon}\right]$;

(ii) $f\left(t, u, u^{\Delta}\right)>\varphi_{p}\left(\frac{b_{i}}{N_{1}^{\prime}}\right)$ for $\left(t, u, u^{\Delta}\right) \in[\xi, T]_{\mathbb{T}} \times\left[b_{i}^{\prime}-\varepsilon, \frac{T}{T-\xi} b_{i}^{\prime}\right] \times\left[0, \frac{b_{i}^{\prime}}{\varepsilon}\right]$;

(iii) $f\left(t, u, u^{\Delta}\right)<\varphi_{p}\left(\frac{a_{i}^{\prime}}{L_{1}^{\prime}}\right)\left(t, u, u^{\Delta}\right) \in[0, T]_{\mathbb{T}} \times\left[0, \frac{T}{T-l} a_{i}^{\prime}\right] \times\left[0, \frac{a_{i}^{\prime}}{\varepsilon}\right]$.

Then problem (1.4) and (1.6) has at least $2 n$ positive solutions.

\section{Example}

In this section, we illustrate an example to explain our results.

EXAMPLE 5.1. Let

$$
\mathbb{T}=\left\{2-\left(\frac{1}{3}\right)^{\mathbb{N}_{0}}\right\} \cup\left\{0, \frac{1}{8}, \frac{1}{4}, \frac{1}{6}, \frac{1}{2}, 1, \frac{5}{4}, \frac{3}{2}, \frac{7}{4}, 2\right\} \cup\left[\frac{1}{20}, \frac{1}{10}\right],
$$

and $T=2$. Consider the following boundary value problem with $p=7$ and $k \in \mathbb{N}_{0}$ : 
(5.1)

$$
\begin{gathered}
\left(\varphi_{p}\left(u^{\Delta}(t)\right)\right)^{\nabla}+\left\{\sum_{k=0}^{7} t^{k}(\rho(t))^{7-k}\right\} t^{\nabla} f\left(t, u(t), u^{\Delta}(t)\right)=0, \quad t \in[0,2]_{\mathbb{T}}, \\
u(0)-4\left(u^{\Delta}\left(\frac{1}{4}\right)+u^{\Delta}(1)\right)=0, \quad u^{\Delta}(2)=0,
\end{gathered}
$$

where

$$
f\left(t, u, u^{\Delta}\right)=\left\{\begin{array}{c}
t+2+\frac{\varepsilon}{10} u^{\Delta}, \quad t \in[0,2]_{\mathbb{T}}, 0 \leq u<80 \text { and } 0 \leq u^{\Delta}<\infty, \\
t+p\left(u, u^{\Delta}\right), \quad t \in[0,2]_{\mathbb{T}}, 80 \leq u<200 \text { and } 0 \leq u^{\Delta}<\infty, \\
t+5 \times 10^{5}+u^{\Delta}, \quad t \in[0,2]_{\mathbb{T}}, 200 \leq u<400 \text { and } 0 \leq u^{\Delta}<\infty, \\
t+s\left(u, u^{\Delta}\right), \quad t \in[0,2]_{\mathbb{T}}, u \geq 400 \text { and } 0 \leq u^{\Delta}<\infty,
\end{array}\right.
$$

here $p\left(u, u^{\Delta}\right)$ and $s\left(u, u^{\Delta}\right)$ satisfy

$$
\begin{gathered}
p\left(80, u^{\Delta}\right)=2+\frac{\varepsilon}{10} u^{\Delta}, \quad p\left(200, u^{\Delta}\right)=5 \times 10^{5}+u^{\Delta}, \quad s\left(400, u^{\Delta}\right)=5 \times 10^{5}+u^{\Delta}, \\
p\left(u, u^{\Delta}\right): \mathbb{R}^{+} \times \mathbb{R}^{+} \rightarrow \mathbb{R}^{+} \text {is continuous, }
\end{gathered}
$$

and

$$
s\left(u, u^{\Delta}\right): \mathbb{R}^{+} \times \mathbb{R}^{+} \rightarrow \mathbb{R}^{+} \text {is continuous. }
$$

If $a_{1}(t)=\left\{\sum_{k=0}^{7} t^{k}(\rho(t))^{7-k}\right\} t^{\nabla}$, then we have $\left(t^{8}\right)^{\nabla}=\left\{\sum_{k=0}^{7} t^{k}(\rho(t))^{7-k}\right\} t^{\nabla}$ [7]. It is easy to see that $\xi_{2}=\eta=1, A=B=4$ and $a_{1}=a_{2}=1$. By taking $r=\frac{3}{2}$, a direct calculation shows that

$$
\begin{aligned}
& M=9\left(\int_{1}^{2}\left\{\sum_{k=0}^{7} t^{k}(\rho(t))^{7-k}\right\} t^{\nabla} \nabla t\right)^{\frac{1}{6}} \approx 22.66, \\
& N=10\left(\int_{0}^{2}\left\{\sum_{k=0}^{7} t^{k}(\rho(t))^{7-k}\right\} t^{\nabla} \nabla t\right)^{\frac{1}{6}} \approx 25.2,
\end{aligned}
$$

and

$$
L=10.5\left(\int_{\frac{3}{2}}^{2}\left\{\sum_{k=0}^{7} t^{k}(\rho(t))^{7-k}\right\} t^{\nabla} \nabla t\right)^{\frac{1}{6}} \approx 25.996 .
$$

When we take $a^{\prime}=2, b^{\prime}=40$ and $c^{\prime}=200$, then

$$
0<a^{\prime}<\frac{L}{N} b^{\prime}<\frac{\eta L}{T N} c^{\prime} .
$$

For $t \in[0,2]_{\mathbb{T}}, 0 \leq u \leq \frac{T b^{\prime}}{\eta}=80$ and $0 \leq u^{\Delta} \leq \frac{b^{\prime}}{\varepsilon}=\frac{40}{\varepsilon}$, we have

$$
f\left(t, u, u^{\Delta}\right)=t+2+\frac{\varepsilon}{10} u^{\Delta}<15.994 \approx \varphi_{p}\left(\frac{b^{\prime}}{N}\right) .
$$

For $t \in[1,2]_{\mathbb{T}}, 200-\varepsilon \leq u \leq \frac{T c^{\prime}}{\eta}=400$ and $200<u^{\Delta} \leq \frac{c^{\prime}}{\varepsilon}+\infty$, we have

$$
f\left(t, u, u^{\Delta}\right)=t+5 \times 10^{5}+u^{\Delta}>4.73 \times 10^{5} \approx \varphi_{p}\left(\frac{c^{\prime}}{M}\right) .
$$

For $t \in\left[\frac{3}{2}, 2\right]_{\mathbb{T}}, 0 \leq u \leq \frac{T a^{\prime}}{r}=2.667$ and $0 \leq u^{\Delta} \leq \frac{a^{\prime}}{\varepsilon}=\frac{2}{\varepsilon}$, we have

$$
f\left(t, u, u^{\Delta}\right)=t+2+\frac{\varepsilon}{10} u^{\Delta}>2.0737 \times 10^{-7} \approx \varphi_{p}\left(\frac{a^{\prime}}{L}\right) .
$$

Consequently, all conditions of Theorem 3.4 are satisfied. By virtue of it, we see that the boundary value problem (5.1) has at least twin positive solutions $u_{1}$ and $u_{2}$ such that

$$
2<\varepsilon \max _{t \in[0,2]_{\mathbb{T}}} u_{1}^{\Delta}(t)+\max _{t \in\left[0, \frac{3}{2}\right]_{\mathbb{T}}} u_{1}(t) \text { with } \varepsilon \max _{t \in[0,2]_{\mathbb{T}}} u_{1}^{\Delta}(t)+\max _{t \in[0,1]_{\mathbb{T}}} u_{1}(t)<40,
$$


and

$$
40<\varepsilon \max _{t \in[0,2]_{\mathbb{T}}} u_{2}^{\Delta}(t)+\max _{t \in[0,1]_{\mathbb{T}}} u_{2}(t) \text { with } \varepsilon \max _{t \in[0,2]_{\mathbb{T}}} u_{2}^{\Delta}(t)+\min _{t \in[1,2]_{\mathbb{T}}} u_{2}(t)<200 .
$$

\section{References}

1. R.P. Agarwal, M. Bohner, P. Rehak, Half-Linear Dynamic Equations, Nonlinear Analysis and Applications: to V. Lakshmikantham on his 80th birthday, vol. 1, Kluwer Acad. Publ. Dordrecht, 2003.

2. B. Aulbach, L. Neidhart, Integration on measure chains, Proceedings of the Sixth International Conference on Difference Equations, CRC, Boca Raton, FL, 2004, pp. 239-252.

3. R.I. Avery, J. Henderson, Two positive fixed points of nonlinear operator on ordered Banach spaces, Comm. Appl. Nonlinear Anal. 8 (2001), 27-36.

4. R.I. Avery, A generalization of the Leggett-Williams fixed point theorem, MSR Hot-Line, 2 (1998), 9-14.

5. F.M. Atici, D.C. Biles, A. Lebedinsky, An application of time scales to economics, Math. Comput. Modelling, 43 (2006), 718-726.

6. M. Bohner, A. Peterson, Advances in Dynamic Equations on Time Scales, Birkhauser, Boston, 2003.

7. M. Bohner, A. Peterson, Dynamic Equations on Time Scales: An Introduction with Applications, Birkhauser, Boston, MA, 2001.

8. V. Jamieson, V. Spedding, Taming nature's numbers, New Scientist: Global Science And Technology Weekly, 2404 (2003), 28-31.

9. Z. He, L. Li, Triple positive solutions for the one-dimensional p-Laplacian dynamic equations on time scales, Math. Comput. Modelling, 45 (2007), 68-79.

10. Z. He, Double positive solutions of three-point boundary value problems for p-Laplacian dynamic equations on time scales, J. Comput. Appl. Math. 182 (2005), 304-315.

11. Z. He, Double positive solutions of three-point boundary value problems for p-Laplacian difference equations, Z. Anal. Anwend. J. Anal. Appl. 24 (2005), 305-315.

12. Z. He, Double positive solutions of boundary value problems for $p$-Laplacian dynamic equations on time scales, Appl. Anal. 84 (2005), 377-390.

13. S. Hong, Triple positive solutions of three-point boundary value problem for p-Laplacian dynamic equations on time scales, J. Comput. Appl. Math. 206 (2007), 967-976.

14. S. Hilger, Ein Maßkettenkalkül mit Anwendung auf Zentrumsmannigfaltigkeiten, Ph.D. Thesis, Universität Würzburg, 1988 (in German).

15. D. Guo, V. Lakshmikantham, Nonlinear Problems in Abstract Cones, Academic press, San Diego, CA, 1988.

16. M. Krasnosel'skii, Positive Solutions of Operator Equations, Noordhoff, Groningen, 1964.

17. V. Lakshmikantham, S. Sivasundaram, B. Kaymakcalan, Dynamic Systems on Measure Chains, Kluwer Academic Publishers, Boston, 1996.

18. R. Leggett, L. Williams, Multiple positive fixed points of nonlinear operators on ordered Banach spaces, Indiana Univ. Math. J. 28 (1979), 673-688.

19. Y. Liu, W. Ge, Twin positive solutions of boundary value problems for finite difference equations with p-Laplacian, J. Math. Anal. Appl. 278 (2003), 551-561.

20. Q. Sheng, M. Fadag, J. Henderson, J.M. Davis, An exploration of combined dynamic derivatives on time scales and their applications, Nonlinear Anal. (RWA) 7 (2006), 395-413.

21. Y.H. Su, B. Li, C. Huang, Positive solution to a singular p-Laplacian BVPs with sign-changing nonlinearity involving derivative on time scales, Adv. Difference Equ. 2009 (2009), 1-21.

22. Y.H. Su, Multiple positive pseudo-symmetric solutions of $p$-Laplacian dynamic equations on time scales, Math. Comput. Modelling, 49 (2009), 1664-1681.

23. Y.H. Su, W.T. Li, H.R. Sun, Triple positive pseudo-symmetric solutions of three-point BVPs for p-Laplacian dynamic equations on time scales, Nonlinear Anal. 68 (2008), 1442-1452.

24. Y.H. Su, W.T. Li, Triple positive solutions of $m$-point BVPs for p-Laplacian dynamic equations on time scales, Nonlinear Anal. 69 (2008), 3811-3820.

25. Y.H. Su, W.T. Li, H.R. Sun, Positive solutions of singular p-Laplacian dynamic equations with sign changing nonlinearity, Appl. Math. Comput. 200 (2008), 352-368.

26. Y.H. Su, W.T. Li, H.R. Sun, Positive solutions of singular p-Laplacian BVPs with sign changing nonlinearity on time scales, Math. Comput. Modelling, 48 (2008), 845-858. 
27. J.P. Sun, Existence of solution and positive solution of BVP for nonlinear third-order dynamic equation, Nonlinear Anal. 64 (2006), 629-636.

28. H.R. Sun, W.T. Li, Existence theory for positive solutions to one-dimensional p-Laplacian boundary value problems on time scales, J. Diff. Equs. 240 (2007), 217-248.

29. H.R. Sun, W.T. Li, Multiple positive solutions for p-Laplacian m-point boundary value problems on time scales, Appl. Math. Comput. 182 (2006), 478-491.

School of Mathematics and Physics, Xuzhou University of Technology, Xuzhou, JIANGSU 221111, CHINA

School of Mathematics and Physics, Xuzhou University of Technology, Xuzhou, JIANGSU 221111, CHINA

Department of Mathematics, University of Texas-Pan American, Edinburg, TX 78539, USA

E-mail address: zsfeng@utpa.edu 